\title{
Metformin alleviates bevacizumab-induced vascular endothelial injury by up-regulating GDF15 and activating the PI3K/AKT/FOXO/ PPAR $\gamma$ signaling pathway
}

\author{
Liqiang Chen ${ }^{1}$, Yajuan Yin ${ }^{2}$, Gang $\operatorname{Liu}^{2} \wedge$ \\ ${ }^{1}$ Cardiovascular Department, The Fourth Hospital of Hebei Medical University, Shijiazhuang, China; ${ }^{2}$ Cardiovascular Department, The First \\ Hospital of Hebei Medical University, Shijiazhuang, China \\ Contributions: (I) Conception and design: G Liu; (II) Administrative support: L Chen; (III) Provision of study materials or patients: Y Yin; (IV) \\ Collection and assembly of data: L Chen; (V) Data analysis and interpretation: L Chen, G Liu; (VI) Manuscript writing: All authors; (VII) Final \\ approval of manuscript: All authors. \\ Correspondence to: Gang Liu, MD. Cardiovascular Department, The First Hospital of Hebei Medical University, No. 89 Donggang Road, \\ Shijiazhuang 050000, China. Email: lugui4235@163.com.
}

Background: Previous studies have reported that the combination of metformin and bevacizumab exhibit favorable efficacy in the treatment of cancer patients, and metformin possesses effects on relieving vascular injury in multiple diseases. Nonetheless, the effect of metformin in alleviating bevacizumab-induced vascular injury remains unknown. Therefore, the present study aimed to investigate the impact of metformin on apoptosis, vascular endothelial injury marker expressions, and inflammation in human umbilical vein endothelial cells (HUVECs), as well as its possible molecular mechanism.

Methods: HUVECs were treated with bevacizumab, metformin or both, and subsequently treated with growth differentiation factor 15 (GDF15) overexpression plasmid, negative control (NC) plasmid, GDF15 small interfering ribonucleic acid (siRNA), NC siRNA, and the phosphoinositide 3-kinase (PI3K) inhibitor LY294002, respectively. After treatment, apoptosis, levels of endothelial injury biomarkers and the potential downstream proteins were detected.

Results: Bevacizumab increased the levels of apoptosis, vascular endothelial injury marker expressions and pro-inflammatory cytokine expressions in HUVECs, while metformin alleviated these effects in bevacizumab-treated HUVECs. Furthermore, GDF15 overexpression reduced the apoptosis, vascular endothelial injury marker expressions, pro-inflammatory cytokine expressions, and activated the PI3K/ protein kinase B (AKT)/forkhead box O (FOXO)/peroxisome proliferator-activated receptor $\gamma(\mathrm{PPAR} \gamma)$ signaling pathway in bevacizumab-treated HUVECs. Subsequently, GDF15 siRNA reduced the effects of metformin on the bevacizumab-induced vascular endothelial injury (as described above) in HUEVCs. Lastly, the PI3K inhibitor exhibited similar effects to those of GDF15 siRNA in bevacizumab-treated HUVECs.

Conclusions: Metformin protected against bevacizumab-induced vascular endothelial injury via activation of GDF15 and the PI3K/AKT/FOXO/PPAR $\gamma$ signaling pathway.

Keywords: Vascular endothelial injury; bevacizumab; metformin; GDF15; PI3K/AKT/FOXO/PPAR $\gamma$ signaling pathway

Submitted Aug 13, 2021. Accepted for publication Oct 02, 2021.

doi: $10.21037 /$ atm-21-4764

View this article at: https://dx.doi.org/10.21037/atm-21-4764

^ ORCID: 0000-0002-7243-0363. 


\section{Introduction}

Despite the considerable treatment progress, cancer is still one of the leading contributors to global mortality. Factors responsible for this not only include the disease itself, but also others such as complications in cancer patients (1). Cancer patients could be accompanied by numerous complications, of which vascular diseases, such as cardiovascular diseases, heart failure, and stroke, are the most harmful (2-4). The majority of vascular endothelial diseases occur during treatment with angiogenesis inhibitors; these agents have been increasingly reported to lead to vascular injury, which is mainly caused by damage to vascular endothelial function $(5,6)$. This situation has led to an increase in research for a combination therapy containing both anti-angiogenic target drugs and drugs that protect against vascular damage.

Bevacizumab, an anti-angiogenic agent broadly applied in cancer therapy, is a monoclonal antibody that specifically targets the combination of vascular endothelial growth factor A (VEGF-A) and its receptors $(7,8)$. Bevacizumab is approved and used in multiple cancers; most commonly cervical cancer, glioblastoma, ovarian cancer, etc., with acceptable efficacy (9-11). Nevertheless, the clinical application of bevacizumab remains an issue, mostly due to bevacizumab-related complications, especially vascularrelated diseases (hypertension, venous thromboembolism, etc.) $(12,13)$. As a targeted drug often used in combination with other anti-tumor therapeutics, it is possible that the addition of other drugs could reduce the injury to vessels induced by bevacizumab. Hence, there is a pressing need for research with this aim.

Metformin has long been the first-line therapeutic agent for type 2 diabetes. However, researchers in the field of oncology have been particularly intrigued by its anti-tumor effect, which has been discovered in recent years (14). A previous randomized controlled study reported that the combined use of metformin with bevacizumab, paclitaxel, and carboplatin shows better survival outcomes in advanced non-small cell lung cancer patients (15). Metformin also exhibits an anti-vascular injury effect in many other diseases via multiple pathways, such as repressing inflammation caused by lipopolysaccharides (LPS) in vascular smooth muscle cells (VSMCs) by modulating peroxisome proliferator activated receptor gamma (PPAR $\gamma$ ) (16). It is also reported that metformin alleviates vascular endothelial injury in sepsis or atherosclerosis $(17,18)$. However, whether the use of metformin is beneficial to the prevention or reduction of bevacizumab-induced vascular endothelial injury in cancer patients has yet to be explored. Besides, numerous studies have disclosed the critical pathways that involve in the regulation of vascular endothelial injury, such as the signal transducer and activator of transcription 3 pathway, the phosphoinositide 3-kinase (PI3K)/protein kinase B (AKT)/ forkhead box $\mathrm{O}$ (FOXO) pathway, transforming growth factor-beta1/Smad pathway, etc. (19-21).

Thus, the aim of the present study is to investigate the impact of metformin on apoptosis, vascular endothelial injury marker expressions, and inflammation in human umbilical vein endothelial cells (HUVECs), as well as its possible molecular mechanism. We present the following article in accordance with the MDAR reporting checklist (available at https://dx.doi.org/10.21037/atm-21-4764).

\section{Methods}

\section{Reagents}

HUVECs (Procell, China; cat.no.: CL-0122; within 36 passages) were cultured in Roswell Park Memorial Institute (RPMI)-1640 medium (Hyclone, USA) containing 10\% fetal bovine serum (FBS) (Tianhang, China). Bevacizumab was obtained from Sinopharm Group Le-Ren-Tang Medicines Co., Ltd. (Sinopharm, China). Metformin and LY294002 were obtained from Med Chem Express Co., Ltd. (MCE, China). The Annexin V-fluorescein isothiocyanate isomer (FITC) Apoptosis Detection Kit was purchased from Beijing Solarbio Science \& Technology Co., Ltd. (Solarbio, China). Trizol reagent was acquired from Thermo Fisher Scientific Inc. (Thermo, USA). HiFiScript genomic deoxyribonucleic acid (DNA) Removal complementary DNA Synthesis Kit and ChemoHS quantitative polymerase chain reaction (qPCR) Mix (None ROX) were purchased from Beijing ComWin Biotech Co., Ltd. (CWBIO, China) and Monad Biotech Co., Ltd. (Monad, China), respectively. E-selectin (CD62E) (KE00169), endothelial-1 (ET-1) (E-CL-H0064), interleukin (IL)-6 (KE00139), thrombomodulin (E-EL-H0166), tumor necrosis factor (TNF)- $\alpha$ (KE00068), and von Willebrand factor (vWF) (E-EL-H2168) enzyme linked immunosorbent assay (ELISA) kits were obtained from Proteintech Group, Inc. (Proteintech, China) and Elabscience Biotechnology Co., Ltd. (Elabscience, China), respectively. The GDF15 overexpression plasmid, negative control (NC) overexpression plasmid, GDF15 small interference ribonucleic acid (siRNA) and NC siRNA were acquired from Shanghai GenePharma Co. Ltd. (GenePharma, China). Lipofectamine ${ }^{\mathrm{TM}} 2000$ 
Transfection Reagent was purchased from Thermo Fisher Scientific (China) Co., Ltd. (Thermo, China). (Radio immunoprecipitation assay) RIPA buffer was obtained from Beijing Solarbio Science \& Technology Co., Ltd. (Solarbio, China). The Western Blotting Luminol Reagent was bought from ZSGB-Bio Co., Ltd. (ZSbio, China).

\section{Bevacizumab and metformin treatment}

For bevacizumab treatment, the HUVECs were incubated with $0.25 \mathrm{mg} / \mathrm{mL}$ bevacizumab (22) for 48 hours (h) at $37{ }^{\circ} \mathrm{C}$. For metformin treatment, the HUVECs were cultured with $50 \mu \mathrm{M}$ metformin (23) for $48 \mathrm{~h}$ at $37{ }^{\circ} \mathrm{C}$. For bevacizumab combined with metformin treatment, the HUVECs were incubated with $50 \mu \mathrm{M}$ metformin plus $0.25 \mathrm{mg} / \mathrm{mL}$ bevacizumab for $48 \mathrm{~h}$. HUVECs that were cultured normally served as the controls. Following incubation, the cells and supernatant were collected for further detection.

\section{Plasmids constructions and transfection}

The GDF15 cDNA (NM_004864.4) was amplified and cloned into pcDNA3.1-kana vector to construct the GDF15 overexpression plasmid. The synthesize nonsense DNA fragment was cloned into pcDNA3.1-kana vector to construct the NC plasmid. The GDF15 or NC overexpression plasmids were transfected into HUVECs with the application of Lipofectamine ${ }^{\mathrm{TM}} 2000$ Transfection Reagent according to the manufacturer's instructions, and the OE-GDF15 or OE-NC cells were then generated. Following transfection, the cells were incubated with bevacizumab for $48 \mathrm{~h}$. Subsequently, the cells and supernatant were collected for followed assessment.

\section{siRNA transfection}

Lipofectamine ${ }^{\mathrm{TM}} 2000$ Transfection Reagent was adopted to transfect the GDF15 or NC siRNA into the HUVECs. Si-GDF 15 and si-NC cells were generated after the transfection. The si-NC and si-GDF15 cells were then treated with bevacizumab alone or bevacizumab plus metformin as described in the "Bevacizumab and metformin treatment" section. Detection of the cells and supernatant was performed after incubation. The target sequences of siRNAs were as follows: GDF15, 5 ' - AAGACTCCAGATTCCGAGAGT-3'; NC, 5'-AGTGAAACAGTGCAGCTG-3'.

\section{LY294002 treatment}

To conduct treatment with LY294002 alone, the HUVECs were treated with $10 \mu \mathrm{M}$ LY294002 (24) for $48 \mathrm{~h}$. For treatment with LY294002, metformin and bevacizumab, the HUVECs were incubated with $50 \mu \mathrm{M}$ metformin, $10 \mu \mathrm{M} \mathrm{LY} 294002$, and $0.25 \mathrm{mg} / \mathrm{mL}$ bevacizumab for $48 \mathrm{~h}$. Meanwhile, the HUVECs incubating with bevacizumab alone or bevacizumab plus metformin were described in the "Bevacizumab and metformin treatment" subsection.

\section{Apoptosis assessment}

The Annexin V-FITC Apoptosis Detection Kit was applied to perform the apoptosis assessment. Briefly, the cells were collected and re-suspended. After incubating with Annexin V-FITC $(5 \mu \mathrm{L})$ and propidium iodide $(\mathrm{PI})(5 \mu \mathrm{L})$ in the dark at room temperature for $30 \mathrm{~min}$, the cells were analyzed with a flow cytometer (BD Biosciences, USA).

\section{ELISA}

The expressions of CD62E, ET-1, TM, vWF, TNF- $\alpha$, and IL-6 in the supernatant were respectively evaluated using CD62E, ET-1, TM, vWF, TNF- $\alpha$, and IL-6 ELISA kits strictly according to the kits' protocols.

\section{Reverse transcription quantitative polymerase chain reaction (RT-qPCR)}

Trizol reagent was applied for RNA extraction. After isolation of the RNA, reverse transcription and qPCR were performed using the HiFiScript gDNA Removal cDNA Synthesis Kit $\left(42^{\circ} \mathrm{C}\right.$ for $15 \mathrm{~min}, 85^{\circ} \mathrm{C}$ for $\left.5 \mathrm{~min}\right)$ and ChemoHS qPCR Mix (None ROX) $\left(95^{\circ} \mathrm{C}\right.$ for $5 \mathrm{~min}$, 1 cycle; $95^{\circ} \mathrm{C}$ for $10 \mathrm{~s}, 61^{\circ} \mathrm{C}$ for $20 \mathrm{~s}, 40$ cycles), respectively. The results were calculated with the $2^{-\Delta \Delta \mathrm{Ct}}$ method and the primers are listed in Table 1.

\section{Western blot}

The cells were harvested and the total protein was extracted using RIPA buffer. After quantification, the thermal denatured protein was separated using 4-20\% precast gel (Beyotime, China) and transferred to a polyvinylidene fluoride membrane (Millipore, USA). After incubation with the diluted primaries $\left(4^{\circ} \mathrm{C}\right.$ overnight) and secondary antibodies $\left(37^{\circ} \mathrm{C}\right.$ for $\left.1 \mathrm{~h}\right)$, the membrane was incubated with 
Table 1 Primers

\begin{tabular}{lll}
\hline Gene & Forward primer $\left(5^{\prime}-3^{\prime}\right)$ & Reverse primer $\left(5^{\prime}-3^{\prime}\right)$ \\
\hline GDF15 & TCAGATGCTCCTGGTGTTGC & CTGGTTAGCAGGTCCTCGTAG \\
PI3K & TCTGTCTCCTCTAAACCCTG & TTCTCCCAATTCAACCAC \\
BCL-2 & TCGCCGAGATGTCCAGC & CCCACCGAACTCAAAGAAGG \\
Bax & TTGCTTCAGGGTTTCATC & ACACTCGCTCAGCTTCTTG \\
PPAR & ACAGGCCGAGAAGGAGA & CAGCGGGAAGGACTTATG \\
$\beta$-actin & GGCACCACACCTTCTACAATGA & GGATAGCACAGCCTGGATAGC \\
\hline
\end{tabular}

Table 2 Antibodies

\begin{tabular}{|c|c|c|c|}
\hline Antibody & Company/country & Catalog number & Dilution rate \\
\hline Rabbit polyclonal to growth differentiation factor 15 (GDF15) & Abcam [United Kingdom (UK)] & ab211364 & $1: 3,000$ \\
\hline Rabbit monoclonal to phosphoinositide 3-kinase (PI3K) & Abcam (UK) & ab32089 & $1: 1,000$ \\
\hline $\begin{array}{l}\text { Rabbit polyclonal to phosphorylated }(p) \text {-protein kinase } \\
\text { B (AKT) }\end{array}$ & Cell Signaling Technology (CST) (USA) & \#9271 & $1: 1,000$ \\
\hline $\begin{array}{l}\text { Rabbit polyclonal to peroxisome proliferator-activated } \\
\text { receptor } \gamma(\text { PPAR } \gamma)\end{array}$ & Proteintech (USA) & 16643-1-AP & $1: 800$ \\
\hline $\begin{array}{l}\text { Rabbit polyclonal to B cell lymphoma-2 }(\mathrm{Bcl}-2) \text { associated } \\
\text { X protein (Bax) }\end{array}$ & Proteintech (USA) & $50599-2-\lg$ & $1: 1,000$ \\
\hline \multicolumn{4}{|l|}{ Secondary antibody } \\
\hline $\begin{array}{l}\text { Goat Anti-Rabbit immunoglobulin G (IgG) heavy- } \\
\text { chain\&light-chain (H\&L) horseradish peroxidase (HRP) }\end{array}$ & ZSbio (China) & ZB-2301 & $1: 10,000$ \\
\hline Goat Anti-Mouse IgG H\&L (HRP) & ZSbio (China) & ZB-2305 & $1: 10,000$ \\
\hline
\end{tabular}

Western Blotting Luminol Reagent to visualize the protein bands. The antibodies are listed in Table 2.

\section{Statistical analysis}

All experiments were replicated three times with three biological replicants. The data in this study were presented as mean \pm standard deviation and analyzed using GraphPad Prism 7.02 (GraphPad Software Inc., USA). One-way analysis of variance (ANOVA) followed by Tukey's multiple comparisons test was used to compare among groups and the unpaired $t$ test was used to compare between two groups. $\mathrm{P}<0.05$ was considered statistically significant.

\section{Results}

Impact of metformin on bevacizumab-induced apoptosis, vascular endothelial injury markers, and inflammation in HUVECs

Bevacizumab treatment increased cell apoptosis in HUVECs, while treatment with metformin reduced cell apoptosis in bevacizumab-treated HUVECs $(\mathrm{P}<0.001)$ (Figure $1 A, 1 B$ ). As for the vascular endothelial injury markers, bevacizumab treatment increased the levels of CD62E (Figure 1C), ET-1 (Figure 1D), TM (Figure 1E), and $\mathrm{vWF}$ (Figure 1F) in HUVECs; however, metformin treatment decreased the levels of these markers (except 


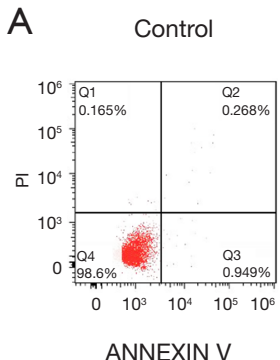

C

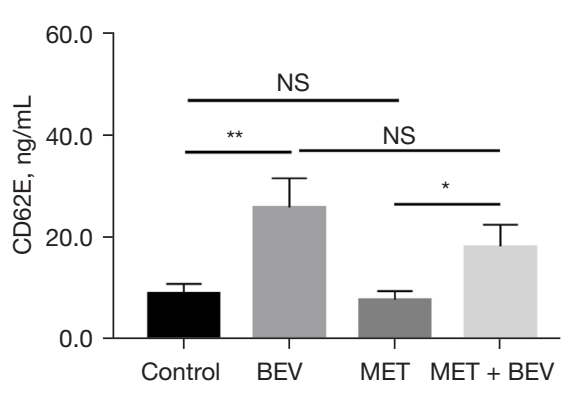

$\mathrm{F}$

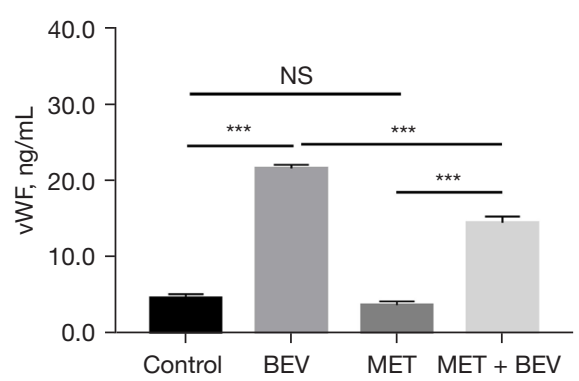

BEV

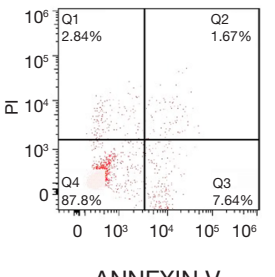

ANNEXIN V

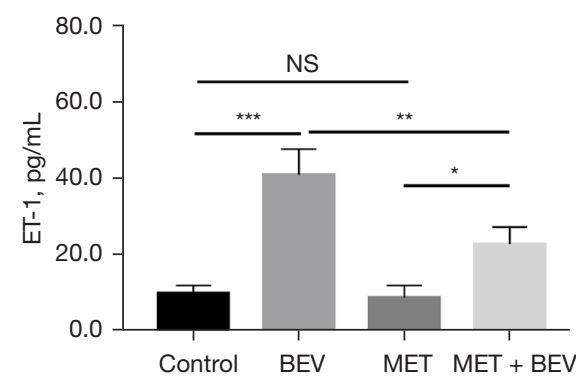

G

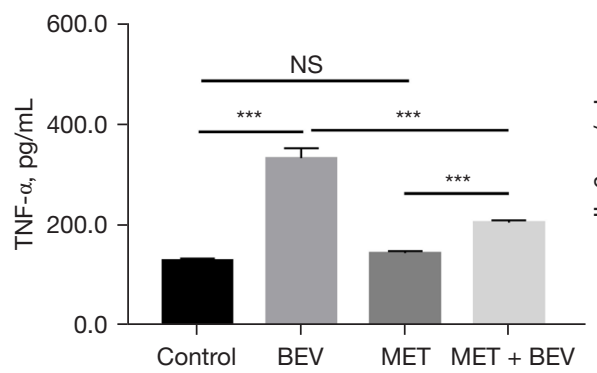

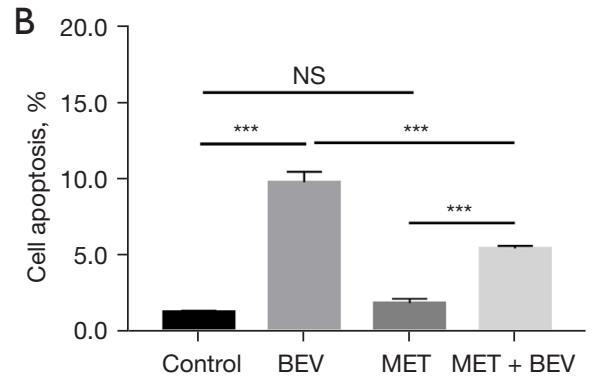

$\mathrm{E}$

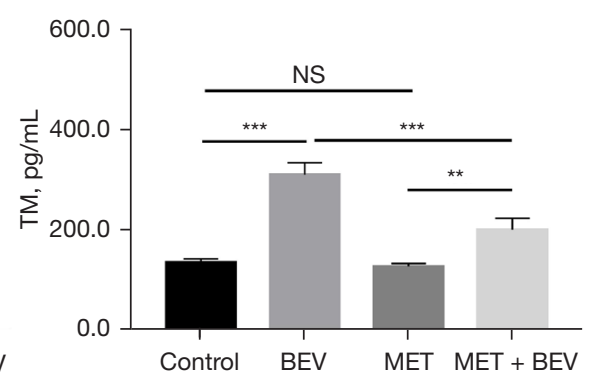

$\mathrm{H}$

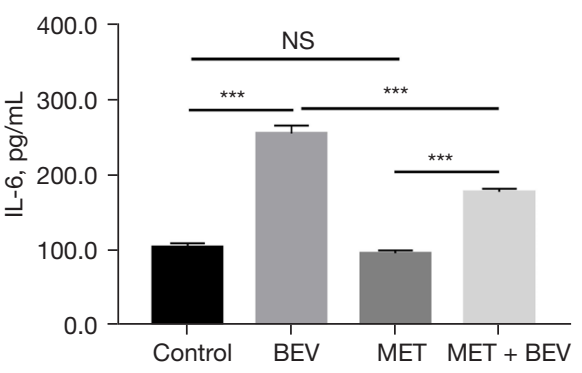

Figure 1 Effect of metformin on bevacizumab-induced apoptosis, vascular endothelial injury markers, and inflammation in HUVECs. Image of flow cytometry of apoptosis (A), cell apoptosis rate (B), CD62E level (C), ET-1 level (D), TM level (E), vWF level (F), TNF- $\alpha$ level (G), and IL-6 level (H) in the control, BEV, MET and MET + BEV groups, respectively. ${ }^{*} \mathrm{P}<0.05$; ${ }^{* *} \mathrm{P}<0.01$; ${ }^{* * *} \mathrm{P}<0.001$; NS, not significant. HUVECs, human umbilical vein endothelial cells, CD62E, selectin E; ET-1, endothelin-1; TM, thrombomodulin; vWF, von Willebrand factor; TNF- $\alpha$, tumor necrosis factor-alpha; IL-6, interleukin-6; BEV, bevacizumab; MET, metformin.

for CD62E) in bevacizumab-treated HUVECs (all $\mathrm{P}<0.05)$. In addition, bevacizumab also increased the expression of TNF- $\alpha$ and IL-6 in HUVECs; however, metformin reduced TNF- $\alpha$ (Figure 1G) and IL-6 (Figure $1 H$ ) expressions in bevacizumab-treated HUVECs (all $\mathrm{P}<0.001)$. The representative images of HUVECs before and after treatment were shown in Figure S1. These results indicated that metformin reduced the bevacizumabinduced apoptosis, vascular endothelial injury markers, and inflammation effects in HUVECs. Subsequently, further experiments revealed that metformin enhanced the GDF15 expression and the activation of the PI3K/AKT/FOXO/ PPAR $\gamma$ signaling pathway in bevacizumab-treated HUVECs (Figure 2A-2F).

\section{Effect of GDF15 overexpression on apoptosis, vascular endothelial injury markers, and inflammation in bevacizumab-treated HUVECs}

GDF15 overexpression reduced cell apoptosis in HUVECs with bevacizumab treatment $(\mathrm{P}<0.01)$ (Figure $3 A, 3 B)$. In terms of vascular endothelial injury markers, GDF15 

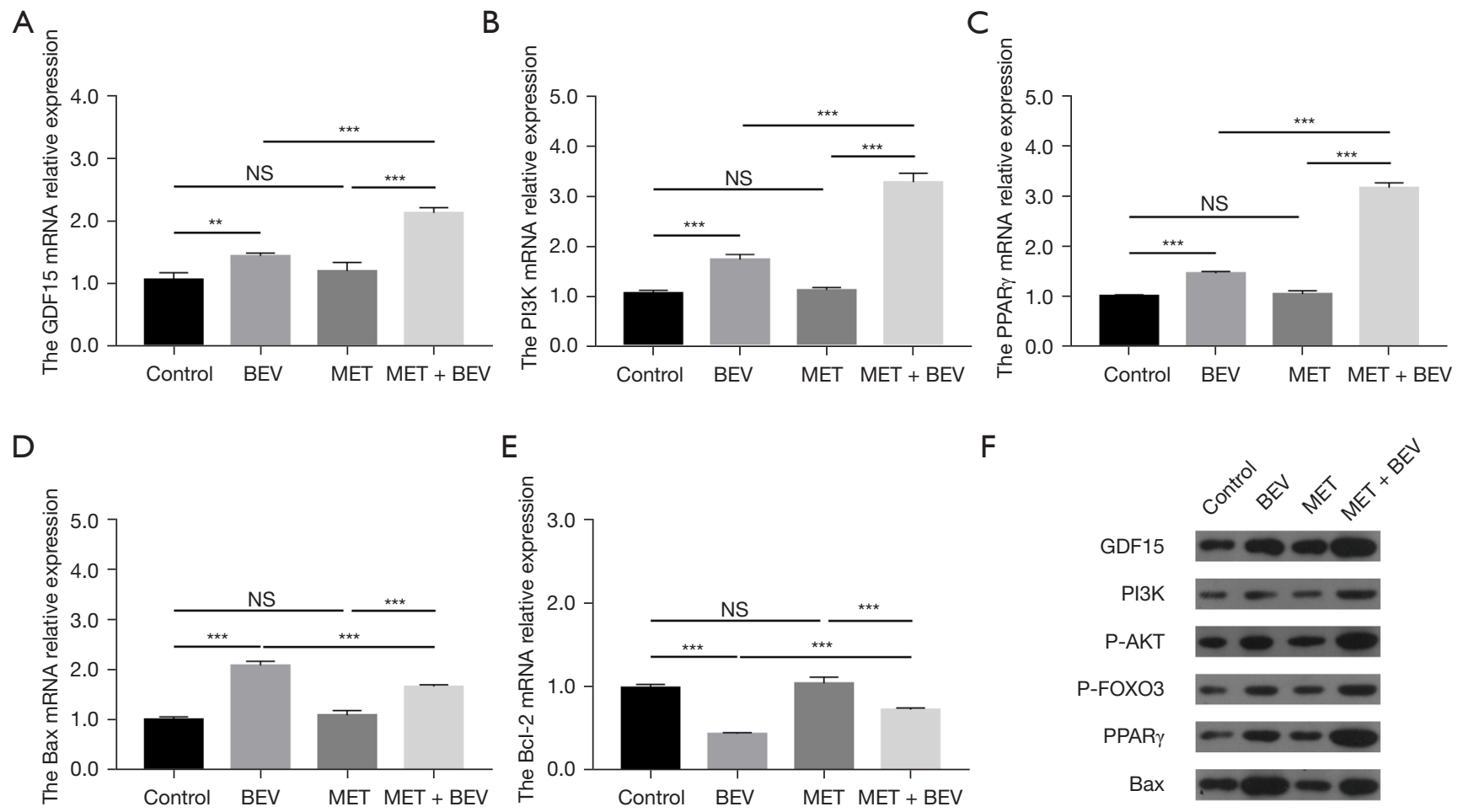

$\mathrm{F}$

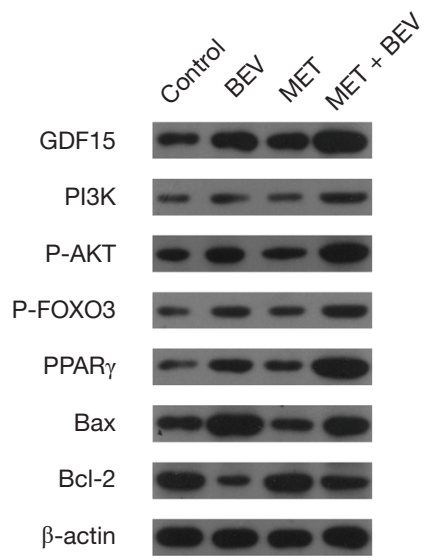

Figure 2 Effect of metformin on GDF15 and PI3K/AKT/FOXO/PPAR $\gamma$ signaling pathway in bevacizumab-treated HUVECs. GDF15 mRNA (A), PI3K mRNA (B), PPAR $\gamma$ mRNA (C), Bax mRNA (D), Bcl-2 mRNA (E) expressions, and protein expressions of GDF15, PI3K, P-AKT, P-FOXO3, PPAR $\gamma, \mathrm{Bax}, \mathrm{Bcl}-2$, and $\beta$-actin (F) in the control, BEV, MET, and MET + BEV groups, respectively. ${ }^{* *} \mathrm{P}<0.01$; ${ }^{* * *} \mathrm{P}<0.001$; NS, not significant. GDF15, growth differentiation factor 15; PI3K, phosphatidylinositol 3-kinase; AKT, threonine kinase; FOXO, forkhead box, sub-group O; P-FOXO3, phosphorylated forkhead box, sub-group O3; PPAR $\gamma$, peroxisome proliferator activated receptor gamma; HUVECs, human umbilical vein endothelial cells; Bax, BCL2 associated X; Bcl-2, B cell lymphoma 2; P-AKT, phosphorylated-threonine kinase; BEV, bevacizumab; MET, metformin.

overexpression inhibited the expressions of CD62E (Figure 3C), ET-1 (Figure 3D), TM (Figure 3E), and vWF (Figure $3 F$ ) in bevacizumab-treated HUVECs (all $\mathrm{P}<0.05$ ). As for inflammation, GDF15 overexpression reduced TNF- $\alpha$ (Figure $3 G$ ) and IL-6 (Figure $3 H$ ) expressions in bevacizumab-treated HUVECs (all $\mathrm{P}<0.01$ ). The GDF15 messenger RNA (mRNA) $(\mathrm{P}<0.001)$ and protein expressions were up-regulated after plasmids transfection; in bevacizumab-treated HUVECs, GFD15 overexpression elevated the mRNA expressions of PI3K, PPAR , and $B$ cell lymphoma-2 (Bcl-2), and decreased the mRNA expression of $\operatorname{Bax}($ all $\mathrm{P}<0.01)$; it also increased the protein expressions of PI3K, P-AKT, P-FOXO3, PPAR $\gamma$, and Bcl-2, and reduced the protein expression of Bax in bevacizumab- treated HUVECs (Figure 4A-4F). Collectively, GDF15 overexpression inhibited bevacizumab-induced apoptosis, vascular endothelial injury markers, and inflammation in HUVECs.

\section{Impact of GDF15 siRNA on bevacizumab-induced apoptosis, vascular endothelial injury markers, and inflammation in HUVECs}

GDF15 siRNA diminished the effect of metformin on mediating apoptosis, as well as the expressions of TM, vWF, TNF- $\alpha$, and IL-6 (all $\mathrm{P}<0.01$ ), but not the expressions of CD62E or ET-1 (all P>0.05) in bevacizumab-treated HUVECs (Figure $5 A-5 H$ ). In addition, GDF15 mRNA 
A

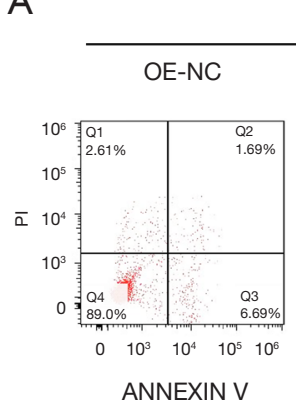

D

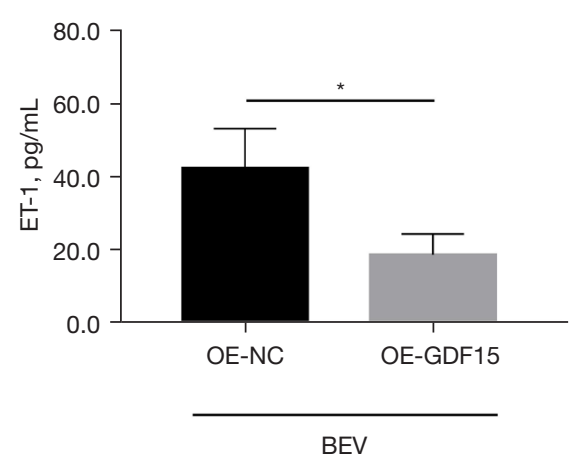

G

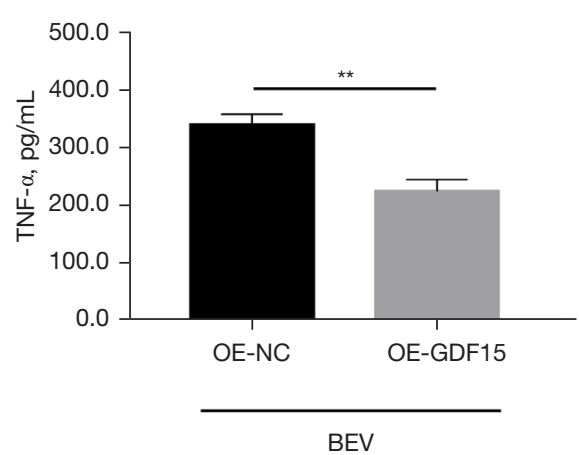

B

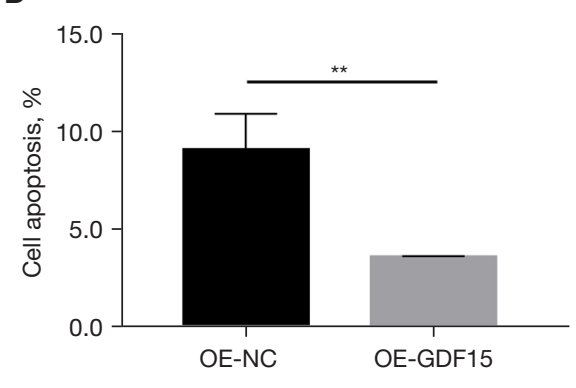

E

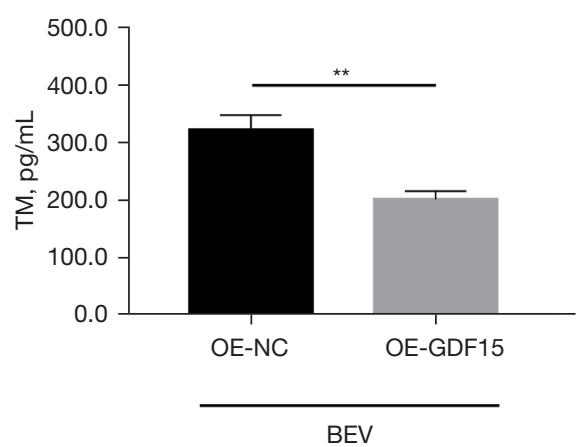

$\mathrm{H}$

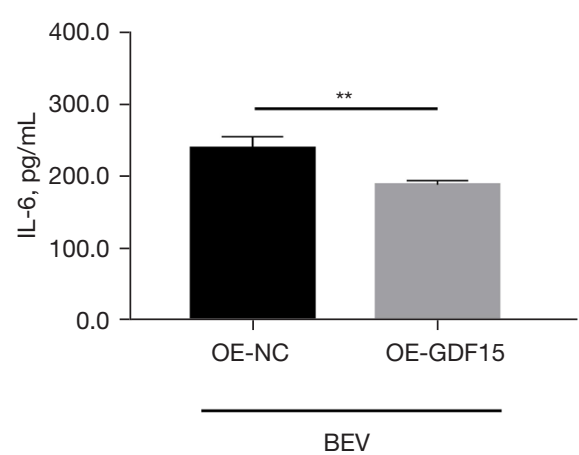

C

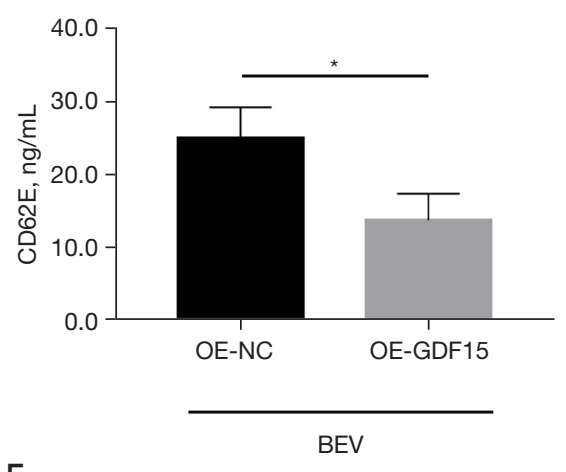

$\mathrm{F}$

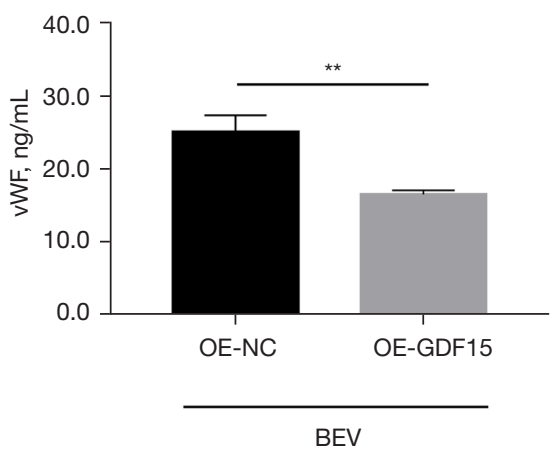

Figure 3 Effect of GDF15 overexpression on apoptosis, vascular endothelial injury markers, and inflammation in bevacizumab-treated HUVECs. Image of flow cytometry of apoptosis (A), cell apoptosis rate (B), CD62E level (C), ET-1 level (D), TM level (E), vWF level (F), TNF- $\alpha$ level (G), and IL-6 level $(\mathrm{H})$ in the OE-NC and OE-GDF15 groups. ${ }^{*} \mathrm{P}<0.05 ;{ }^{*} \mathrm{P}<0.01$. GDF15, growth differentiation factor 15; HUVECs, human umbilical vein endothelial cells; CD62E, selectin E; ET-1, endothelin-1; TM, thrombomodulin; vWF, von Willebrand factor; TNF- $\alpha$, tumor necrosis factor-alpha; IL-6, interleukin-6; OE, overexpression; NC, negative control; BEV, bevacizumab.

relative expression (all $\mathrm{P}<0.001$ ) and protein expression was down-regulated post-siRNA transfection; moreover, GDF15 siRNA decreased the effect of metformin on PI3K, $P P A R \gamma, B c l-2$, and Bax mRNA expressions in bevacizumabtreated HUVECs (all $\mathrm{P}<0.001$ ); in addition, the protein expressions of PI3K, PPAR $\gamma, \mathrm{Bax}, \mathrm{Bcl}-2$, and $\beta$-actin exhibited a similar trend as described above, and P-AKT as well as $\mathrm{P}-\mathrm{FOXO} 3$ protein expressions also displayed similar trends (Figure 6A-6F). These results implied that GDF15 inhibition reduced the effects of metformin on bevacizumab-induced apoptosis, vascular endothelial injury markers, inflammation, and PI3K/AKT/FOXO/PPAR $\gamma$ 


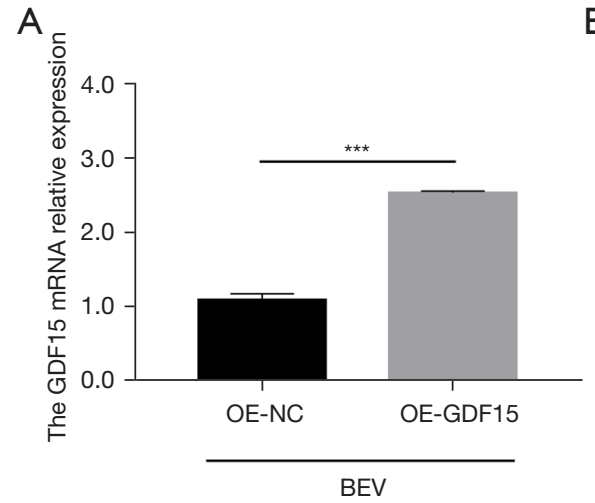

D

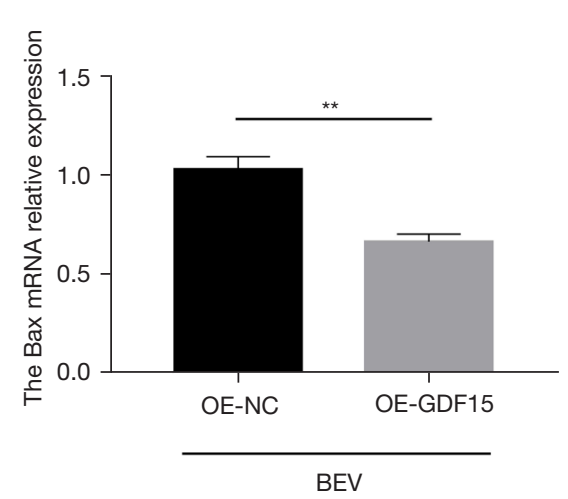

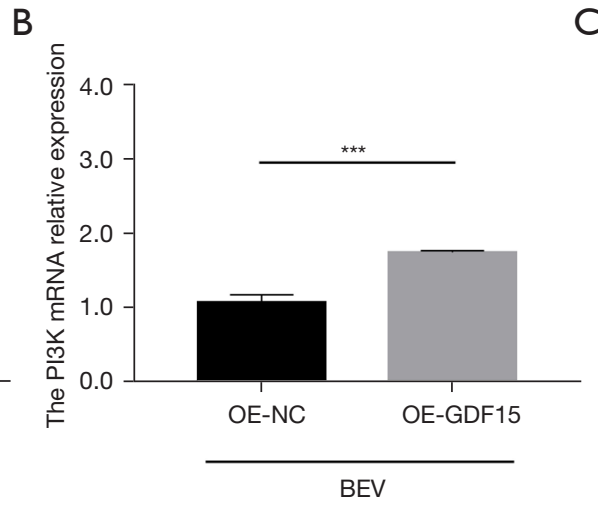

E

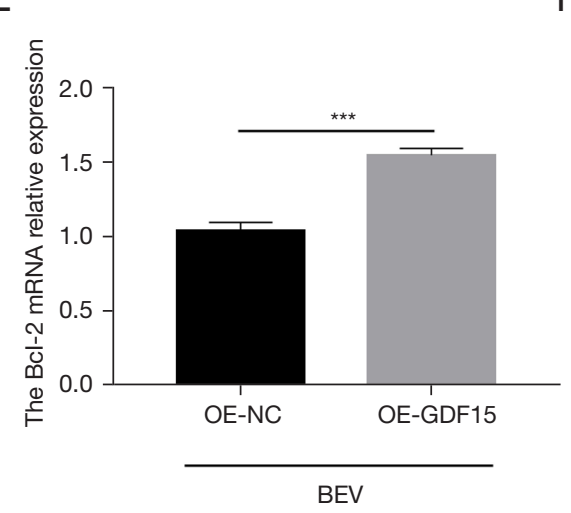

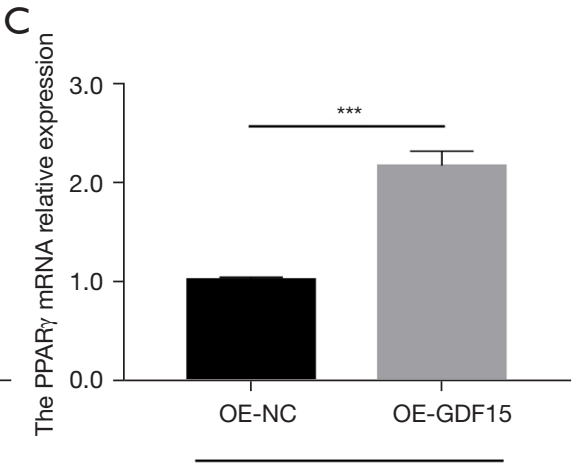

BEV

$\mathrm{F}$

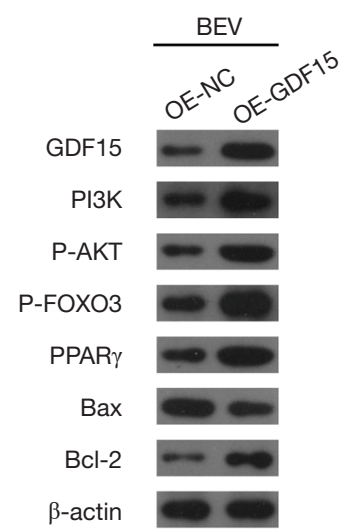

Figure 4 Effect of GDF15 overexpression on the PI3K/AKT/FOXO/PPAR $\gamma$ signaling pathway in bevacizumab-treated HUVECs. GDF15 mRNA (A), PI3K mRNA (B), PPAR $\gamma$ mRNA (C), Bax mRNA (D), Bcl-2 mRNA (E), and protein expressions of GDF15, PI3K, P-AKT, $\mathrm{P}-\mathrm{FOXO} 3, \mathrm{PPAR} \gamma, \mathrm{Bax}, \mathrm{Bcl}-2$, and $\beta$-actin $(\mathrm{F})$ in the OE-NC and OE-GDF15 groups, respectively. ${ }^{* *} \mathrm{P}<0.01 ;{ }^{* * *} \mathrm{P}<0.001$. GDF15, growth differentiation factor 15; PI3K, phosphatidylinositol 3-kinase; AKT, threonine kinase; FOXO, forkhead box, sub-group O; PPAR $\gamma$, peroxisome proliferator activated receptor gamma; HUVECs, human umbilical vein endothelial cells; Bax, BCL2 associated X; Bcl-2, B cell lymphoma 2; P-AKT, phosphorylated-threonine kinase; P-FOXO3, phosphorylated-forkhead box, sub-group O3; OE, overexpression; NC, negative control; BEV, bevacizumab.

signaling pathway activation in HUVECs.

\section{Impact of the PI3K inhibitor on bevacizumab-induced apoptosis, vascular endothelial injury markers, and inflammation in HUVECs}

The PI3K inhibitor reduced the effects of metformin on ET-1, TM, vWF, TNF- $\alpha$, and IL-6 expressions in bevacizumab-treated HUVECs (all $\mathrm{P}<0.05$ ), but did not reduce cell apoptosis or CD62E expression (all $\mathrm{P}>0.05$ ) (Figure $7 A-7 H$ ).

Additionally, the PI3K inhibitor down-regulated PI3K, $P P A R \gamma$, and $B c l-2$ mRNA expressions and up-regulated Bax mRNA expression (all $\mathrm{P}<0.01$ ); however, it did not alter the
mRNA expression of GDF15 ( $\mathrm{P}>0.05)$ in bevacizumab and metformin-treated HUVECs; as for protein expressions, the PI3K inhibitor decreased PI3K, P-AKT, P-FOXO3, $\mathrm{PPAR} \gamma$, and $\mathrm{Bcl}-2$ protein expressions, increased the protein expression of Bax, and did not alter the protein expression of GDF15 (Figure $8 A-8 F$ ). Together, these results indicated that the PI3K inhibitor diminished the effects of metformin on bevacizumab-induced cell apoptosis, vascular endothelial injury markers, and inflammation in HUVECs.

\section{Discussion}

Bevacizumab-induced vascular complications, mostly derived from vascular endothelial injury, have become a major issue 
A

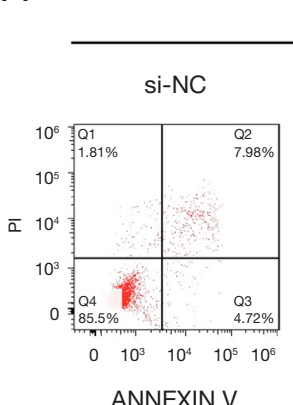

C

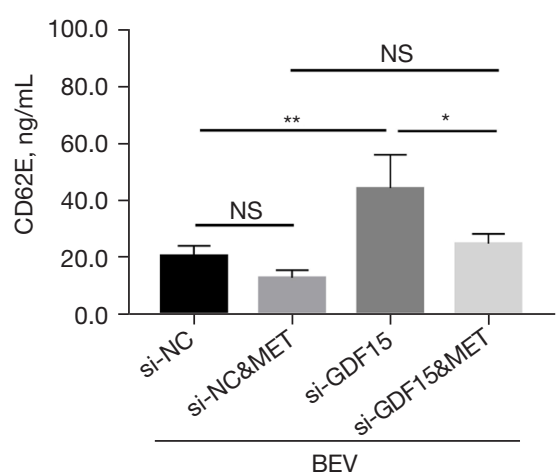

$\mathrm{F}$

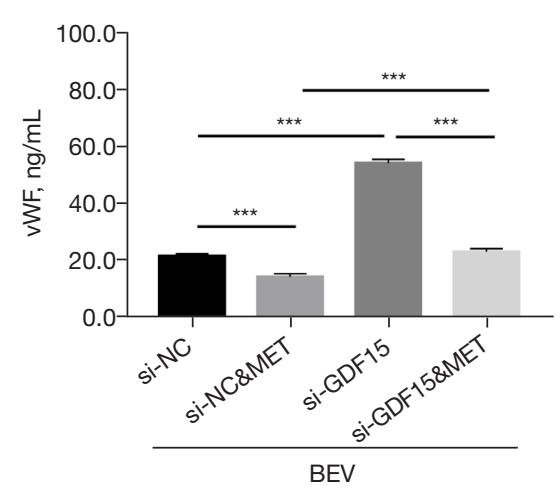

BEV
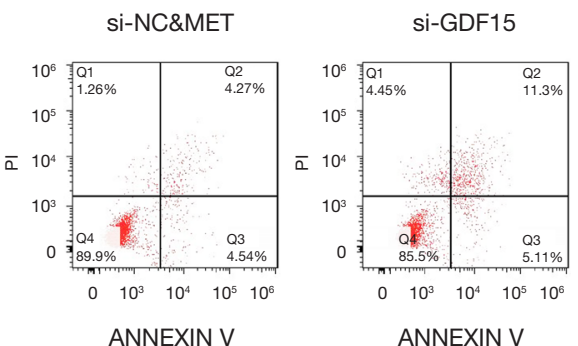

ANNEXIN V
B
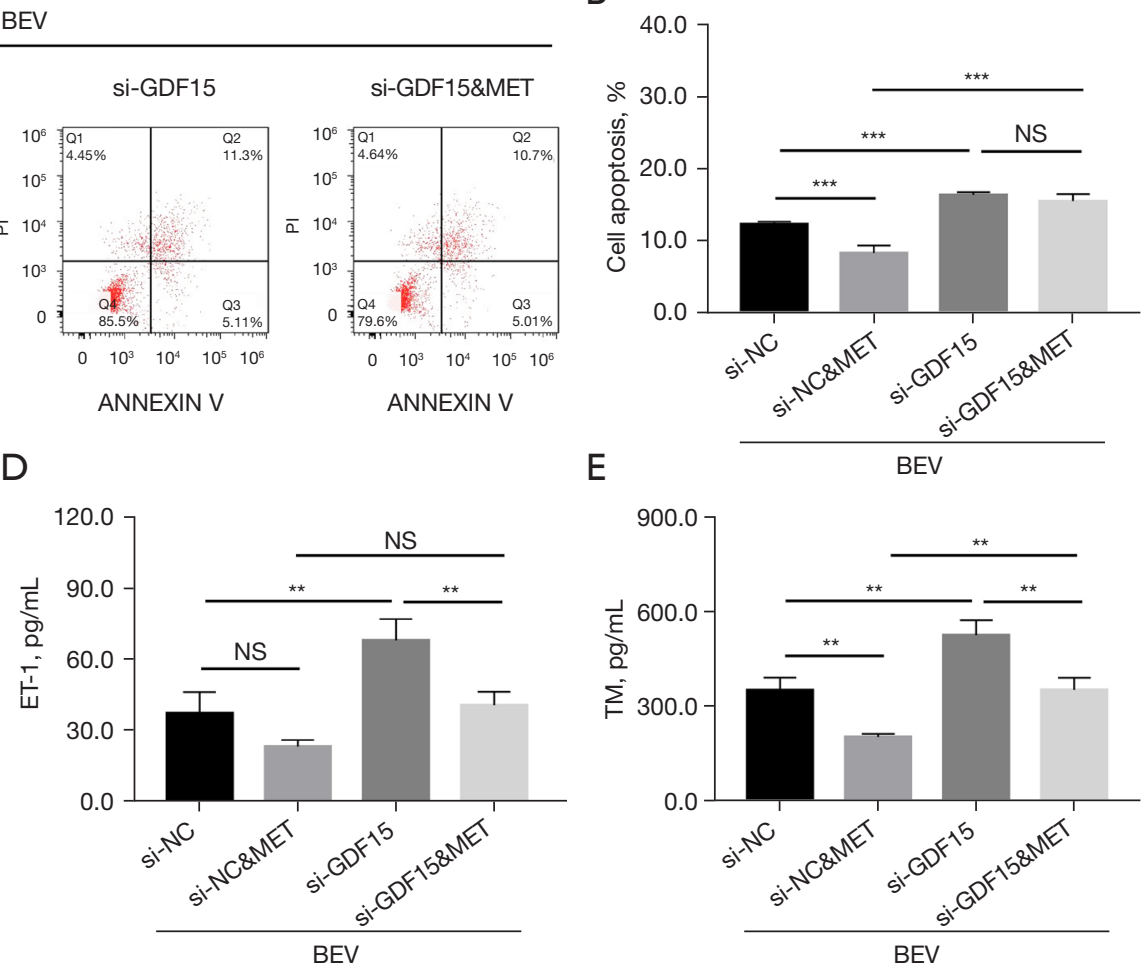

G

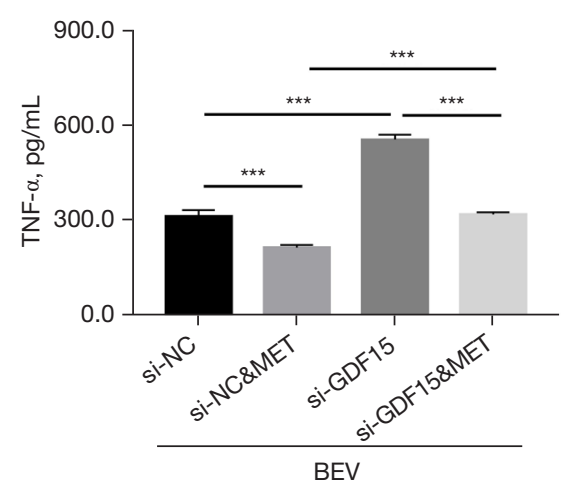

$\mathrm{E}$

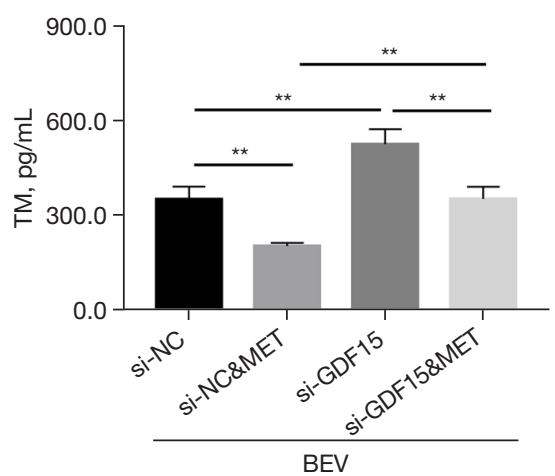

$\mathrm{H}$

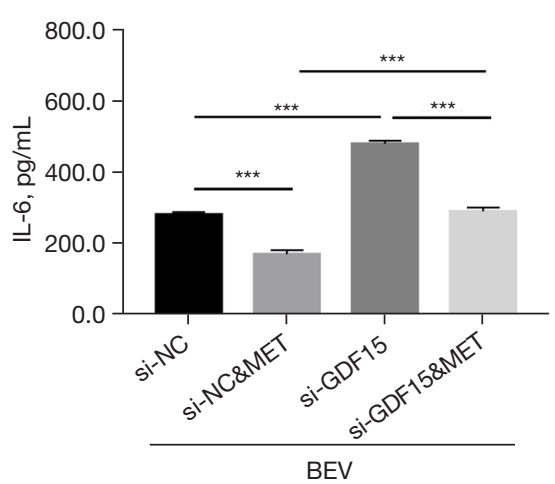

Figure 5 Effect of GDF15 siRNA on apoptosis, vascular endothelial injury markers, and inflammation in bevacizumab and metformintreated HUVECs. Image of flow cytometry of apoptosis (A), cell apoptosis rate (B), CD62E level (C), ET-1 level (D), TM level (E), vWF level (F), TNF- $\alpha$ level (G), and IL-6 level (H) in the si-NC, si-NC\&MET, si-GDF15, and si-GDF15\&MET groups, respectively. ${ }^{*}<0.05$; ${ }^{*} \mathrm{P}<0.01 ;{ }^{* *} \mathrm{P}<0.001$; NS, not significant. GDF15, growth differentiation factor 15 ; HUVECs, human umbilical vein endothelial cells; CD62E, selectin E; ET-1, endothelin-1; TM, thrombomodulin; vWF, von Willebrand factor; TNF- $\alpha$, tumor necrosis factor-alpha; IL-6, interleukin-6; si, siRNA; NC, negative control; MET, metformin; BEV, bevacizumab.

to manage in addition to treating the cancer itself (25). For example, a prior study revealed that in patients with metastatic breast cancer or metastatic colorectal cancer, compared to patients receiving conventional chemotherapy, only patients receiving conventional chemotherapy combined with bevacizumab display cardiovascular and thromboembolic events. In these patients, the number of patients who develop acute myocardial infarction, coronary artery disease, and thromboembolic event are $14.81 \%, 19.23 \%$, and $17.86 \%$, respectively (26). Additionally, a retrospective cohort study 

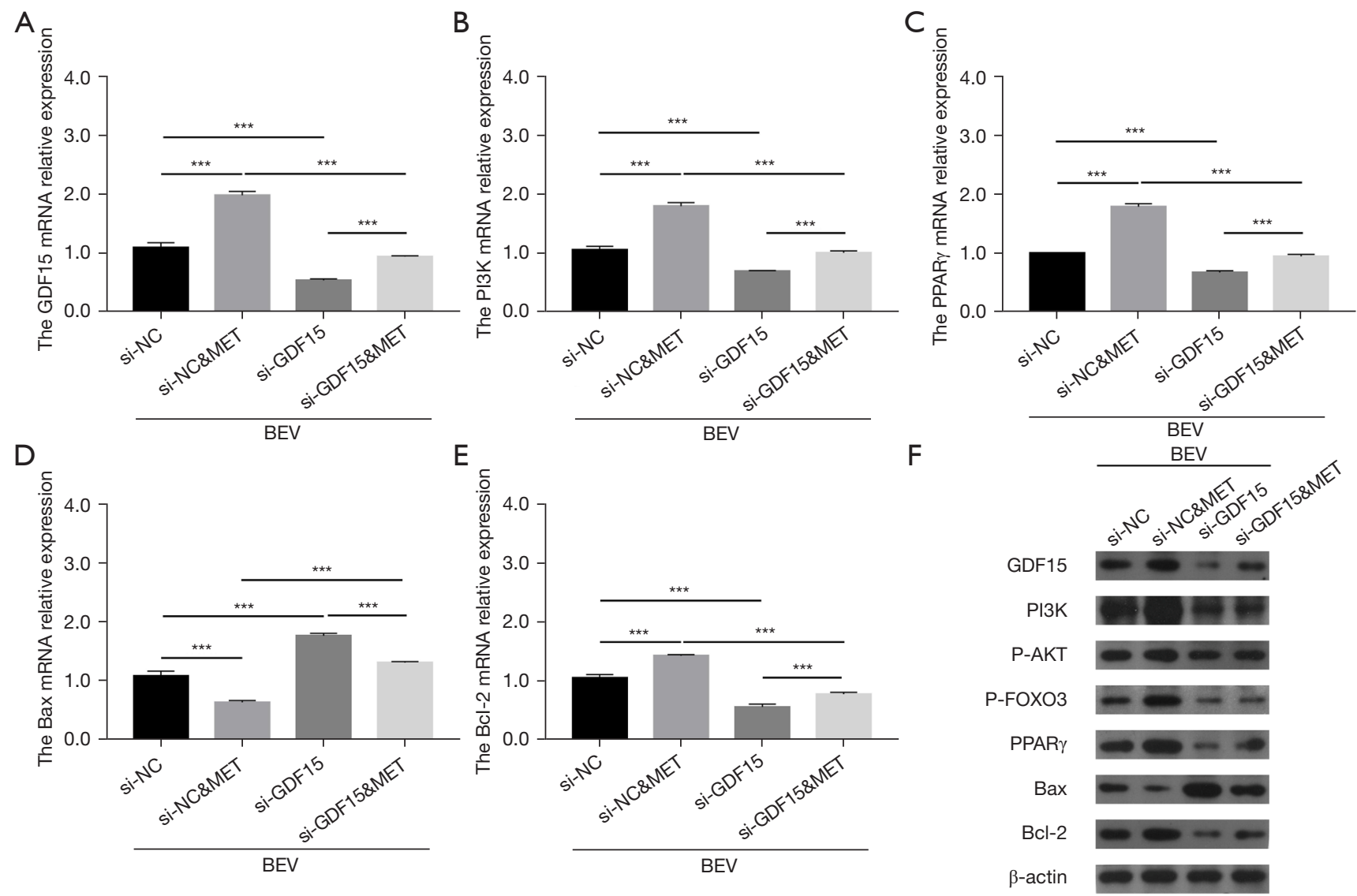

Figure 6 Effect of GDF15 siRNA on the PI3K/AKT/FOXO/PPAR $\gamma$ signaling pathway in bevacizumab and metformin-treated HUVECs. GDF15 mRNA (A), PI3K mRNA (B), PPAR $\gamma$ mRNA (C), Bax mRNA (D), Bcl-2 mRNA (E), and protein expressions of GDF15, PI3K, P-AKT, P-FOXO3, PPAR $\gamma$, Bax, Bcl-2, and $\beta$-actin (F) in the si-NC, si-NC\&MET, si-GDF15, si-GDF15\&MET groups, respectively. ${ }^{* * *} \mathrm{P}<0.001$. GDF15, growth differentiation factor 15; PI3K, phosphatidylinositol 3-kinase; AKT, threonine kinase; FOXO, forkhead box, sub-group O; PPAR $\gamma$, peroxisome proliferator activated receptor gamma; HUVECs, human umbilical vein endothelial cells; Bax, BCL2 associated X; Bcl-2, B cell lymphoma 2; P-AKT, phosphorylated- threonine kinase; P-FOXO3, phosphorylated-forkhead box, sub-group $\mathrm{O} 3$; OE, overexpression; NC, negative control; BEV, bevacizumab.

reported that in cancer patients (multiple types) treated with bevacizumab, 28 out of 230 patients were re-treated in hospital due to major cardiovascular-related events (27). Another study showed that in patient-derived cervical cancer xenograft models, bevacizumab treatment reduces abnormal microvessels but results in worse perfusion and hypoxic damage, suggesting a possible injury to the overall vascular system (28). Nevertheless, there are still no validated solutions to bevacizumab-induced vascular endothelial injury.

Several theories regarding the potential solutions exist, including the combination with metformin, which has been shown to be a protector of vascular functions, especially for cancer patients complicated with diabetes mellitus.
For instance, a previous study illustrated that metformin enhances the recovery of peripheral blood flow in patients with peripheral atherosclerosis (29). In addition, another study revealed that the endothelial inflammation caused by high fat can be reduced by treatment with metformin and Abelmoschus esculentus by increasing the microRNA 146a (miR-146a) and miR-155 levels in vivo and in vitro (30).

According to several previous reports, metformin has been shown to protect the vascular function of cancer patients. One study demonstrated that metformin reduces the processes related tumor progression and enhances chemosensitivity by promoting vessel normalization in metastatic breast cancer in vivo and in vitro (31). However, 
A

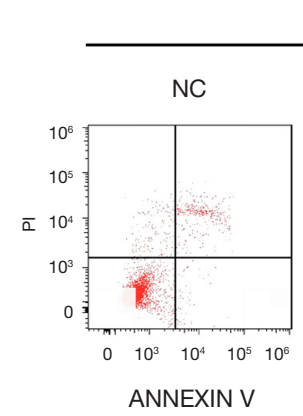

C

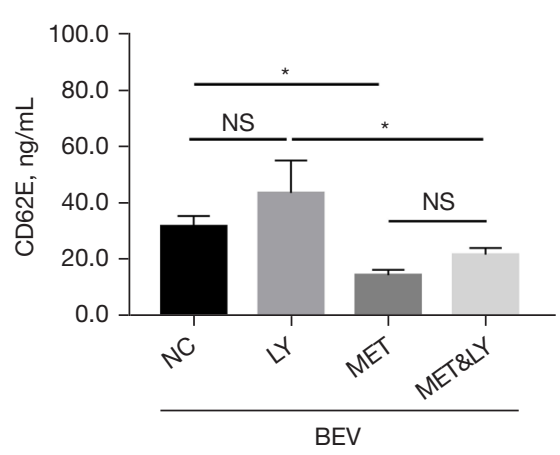

$\mathrm{F}$

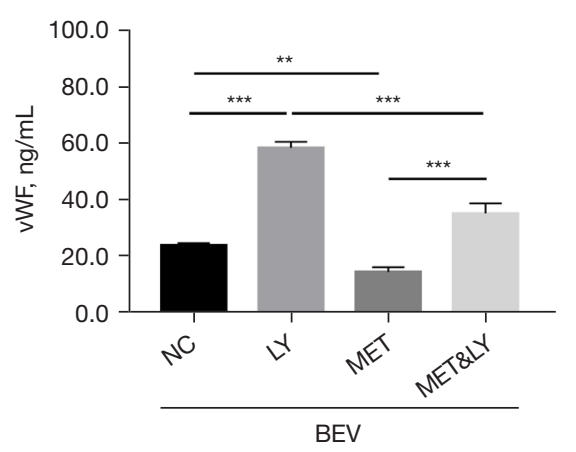

BEV

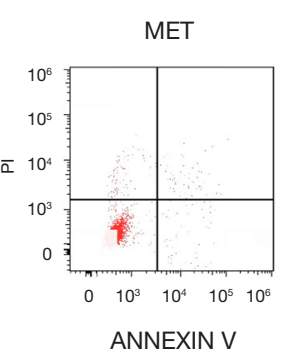

$\mathrm{D}$

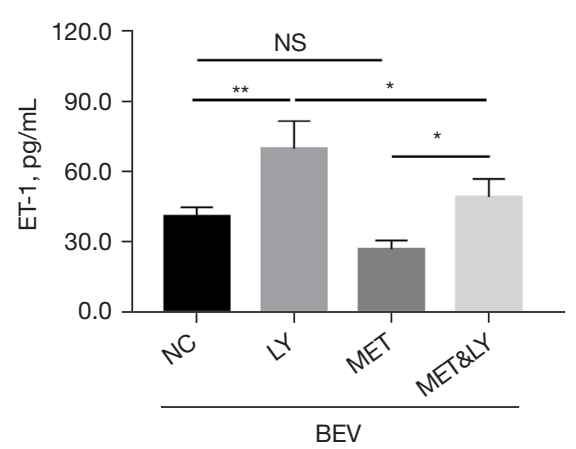

G

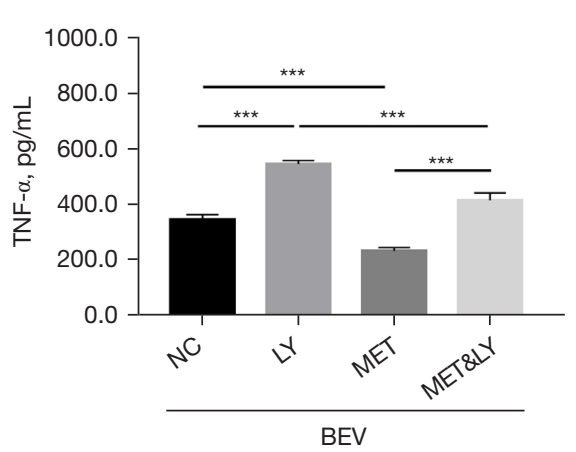

B

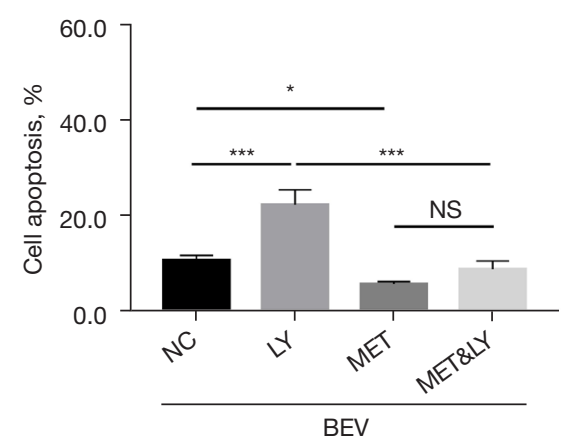

E

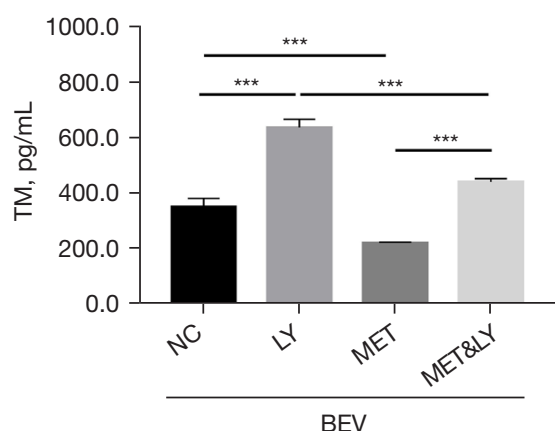

$\mathrm{H}$

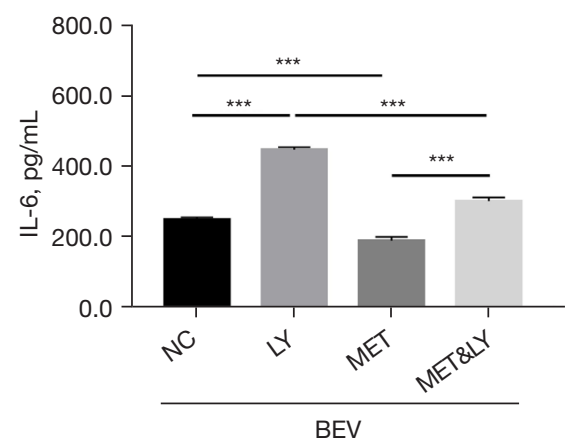

Figure 7 Effect of the PI3K inhibitor on apoptosis, vascular endothelial injury markers, and inflammation in bevacizumab and metformintreated HUVECs. Image of flow cytometry of apoptosis (A), cell apoptosis rate (B), CD62E level (C), ET-1 level (D), TM level (E), vWF level (F), TNF- $\alpha$ level (G), and IL-6 level (H) in the NC, LY, MET, MET\&LY groups, respectively. ${ }^{*} \mathrm{P}<0.05 ;{ }^{* *} \mathrm{P}<0.01 ;{ }^{* * *} \mathrm{P}<0.001$; NS, not significant. PI3K, phosphatidylinositol 3-kinase; HUVECs, human umbilical vein endothelial cells; CD62E, selectin E; ET-1, endothelin-1; TM, thrombomodulin; vWF, von Willebrand factor; TNF- $\alpha$, tumor necrosis factor-alpha; IL-6, interleukin-6; NC, negative control; LY, LY294002; MET, metformin; BEV, bevacizumab.

to the best of our knowledge, the potential effect of metformin in relieving bevacizumab-induced vascular endothelial injury was evaluated for the first time in our study. In this study, we found that metformin treatment reduced the effect of bevacizumab in inducing apoptosis, vascular endothelial injury markers, and inflammation in
HUVECs. There were some probable explanations for these findings. First, metformin has been elucidated to possess the ability to regulate vascular endothelial function in a protective way, which is achieved through eliminating inflammation and hypoxia damage or promoting angiogenic behaviors, and so on, via regulation of multiple factors 

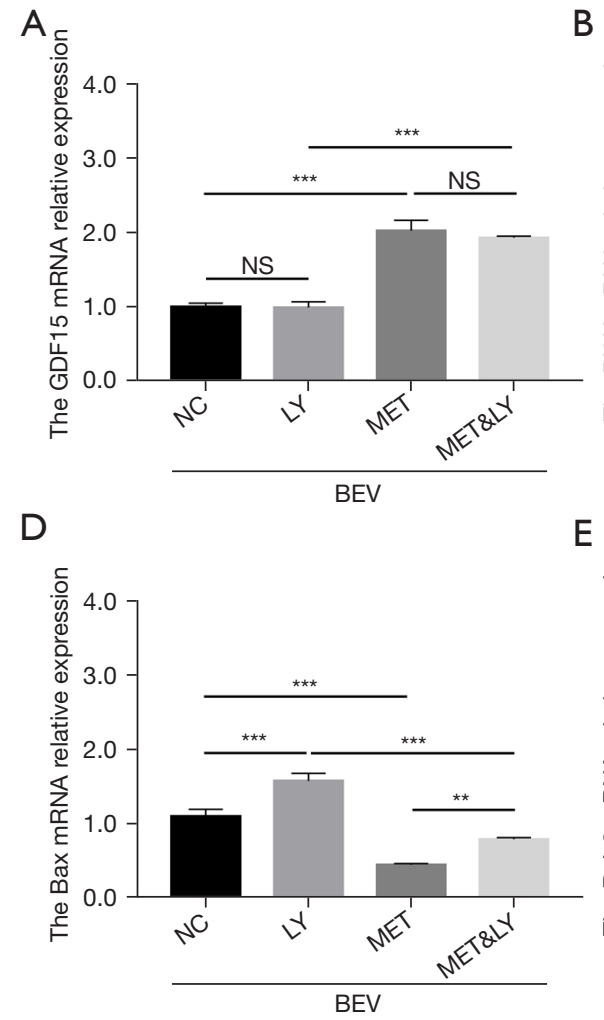

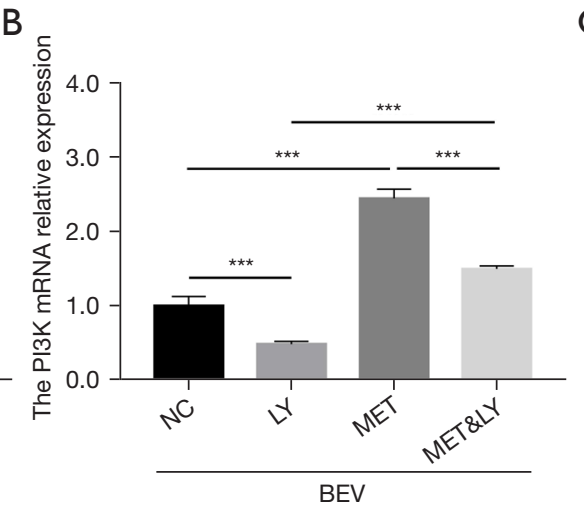

E

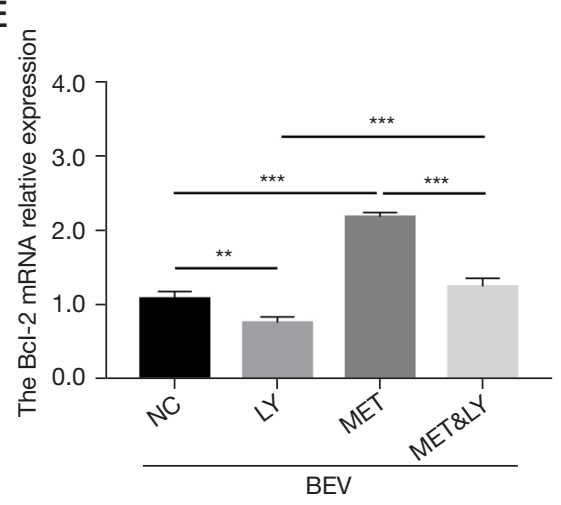

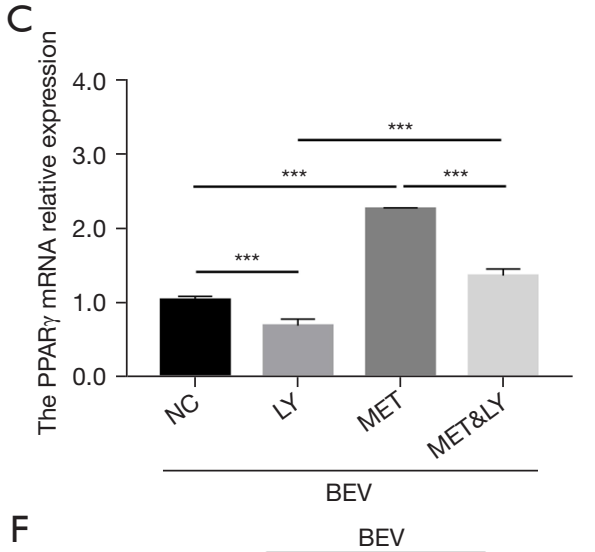

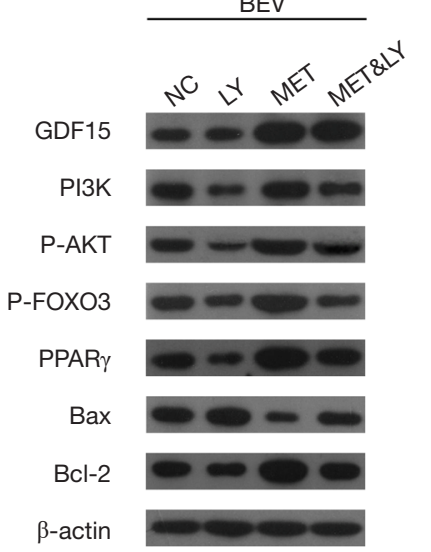

Figure 8 Effect of the PI3K inhibitor on GDF15 and PI3K/AKT/FOXO/PPAR $\gamma$ signaling in bevacizumab and metformin-treated HUVECs. GDF15 mRNA (A), PI3K mRNA (B), PPAR $\gamma$ mRNA (C), Bax mRNA (D), Bcl-2 mRNA (E), and protein expressions of GDF15, PI3K, P-AKT, P-FOXO3, PPAR $\gamma$, Bax, Bcl-2, and $\beta$-actin (F) in the NC, LY, MET and MET\&LY groups, respectively. **P<0.01; ${ }^{* * *} \mathrm{P}<0.001$; NS, not significant. PI3K, phosphatidylinositol 3-kinase; AKT, threonine kinase; FOXO, forkhead box, sub-group O; PPAR $\gamma$, peroxisome proliferator activated receptor gamma; Bax, BCL2 associated X; Bcl-2, B cell lymphoma 2; P-AKT, phosphorylatedthreonine kinase; P-FOXO3, phosphorylated- forkhead box, sub-group O 3; NC, negative control; LY, LY294002; MET, metformin; BEV, bevacizumab.

(such as (adenosine 5'-monophosphate-activated protein kinase) AMPK, vascular endothelial growth factor receptor (VEGFR), hedgehog pathway, etc.) (17,23,32). Also, as revealed by our further experiments, metformin might also reduce bevacizumab-induced vascular endothelial injury via activation of GDF15 expression and the PI3K/ AKT/FOXO/PPAR $\gamma$ signaling pathway in HUVECs. Therefore, our study showed that metformin could reduce bevacizumab-induced vascular endothelial injury in HUVECs.

GDF15 and the PI3K/AKT/FOXO/PPAR $\gamma$ signaling pathway are both involved in the mechanisms through which metformin functions in human body. In terms of GDF15, a previous study revealed that oral metformin elevates GDF15 serum expression, and also decreases food intake, body mass, fasting insulin level, and glucose intake in mice with a high-fat diet; however, these effects are all inhibited in GDF15-null mice (33). A secondary analysis (from a genome-wide association study) showed that upregulated GDF15 is correlated with decreased risk of coronary artery disease (34). As for the PI3K/AKT/PPAR $\gamma$ signaling pathway, one study elucidated that metformin represses endometrial cancer cell proliferation and migration by mediating the PI3K/AKT/MDM2 signaling pathway (35). In addition, another study revealed that in vascular smooth muscle cells, metformin decreases the inflammatory responses resulting from LPS treatment by modulating Toll-like receptor 4 (TLR4) and PPAR $\gamma(16)$. 
Another study demonstrated that metformin treatment alleviates cell senescence and apoptosis by repressing hyperglycaemia sirtuin 1 (SIRT1) decrease and expressions of its downstream targets, including FOXO- 1 and p53/p21, in mouse microvascular endothelial cells (36).

Most importantly, GDF 15 and the PI3K/AKT/ FOXO/PPAR $\gamma$ signaling pathway are also involved in the regulation of vascular endothelial function; although not all in cancer patients, these previous findings still provide valuable information regarding their roles in vascular diseases. For example, one previous study showed that Epac agonist improves endothelial cell survival by enhancing the activation of $\mathrm{PI} 3 \mathrm{~K} / \mathrm{AKT}$ and mitogen-activated protein kinase (MEK)/extracellular signal-regulated kinase (ERK) signaling pathways (37). Additionally, the apolipoprotein $M$ and sphingosine-1-phosphate complex reduces TNF$\alpha$-induced endothelial cell injury and inflammation in HUVECs via the PI3K/AKT signaling pathway (38). Another study revealed that PPAR $\gamma$ can interact with ubiquitin protein ligase $\mathrm{E} 3$ component $\mathrm{N}$-recognin 5 (UBR5)/ATM interactor (ATMIN), and subsequently enhances the maintenance of homeostasis in endothelial cells (39). An in vitro study demonstrated that senescent cell-produced GDF15 promotes the function of vascular progenitor cells in endothelial colony forming cells derived from adult blood (40). Another in vivo and in vitro study showed that AKT1 increases the recovery of stimuliinduced endothelial-barrier via mediating tight-junction protein turnover, which is modulated by FOXO (41). An interesting previous study revealed that FOXO3 is involved in the regulation of vascular endothelial injury, and our results were consistent with that previous study (42).

Our study found that metformin treatment reduced bevacizumab-induced apoptosis, vascular endothelial injury markers, and inflammation in HUVECs by enhancing GDF15 expression and activating the PI3K/AKT/ FOXO/PPAR $\gamma$ signaling pathway. Firstly, these factors, including GDF15 and the PI3K/AKT/FOXO/PPAR $\gamma$ signaling pathway, are all involved in the mechanism of action of metformin. In addition, they also participate in the regulation of endothelial functions; hence, our study indicates that metformin could reduce endothelial damage by regulating GDF 15 and PI3K/AKT/FOXO/PPAR $\gamma$ signaling pathway (37-41). To the best of our knowledge, this was the first study assessing the protective role and potential regulatory factors of metformin in bevacizumabinduced vascular endothelial injury in HUVECs. However, more experiments are required to validate our findings, especially regarding the mechanisms identified in this study.

In conclusion, metformin protects against bevacizumabinduced vascular endothelial injury and inflammation via activation of GDF15 and the PI3K/AKT/FOXO/PPAR $\gamma$ signaling pathway.

\section{Acknowledgments}

Funding: This study was supported by The Medical Science Research Key Project of Hebei Province, China (No. 20211599), Project of Hebei Provincial Department of Finance, China (No. LS201808), Natural Science Foundation of Hebei Province, China (No. H2019206715) and Department School Consultation Fund - Science and Technology Innovation Project, China (No. 2020TXJC06).

\section{Footnote}

Reporting Checklist: The authors have completed the MDAR reporting checklist. Available at https://dx.doi. org/10.21037/atm-21-4764

Data Sharing Statement: Available at https://dx.doi. org/10.21037/atm-21-4764

Conflicts of Interest: All authors have completed the ICMJE uniform disclosure form (available at https://dx.doi. org/10.21037/atm-21-4764). The authors have no conflicts of interest to declare.

Ethical Statement: The authors are accountable for all aspects of the work in ensuring that questions related to the accuracy or integrity of any part of the work are appropriately investigated and resolved.

Open Access Statement: This is an Open Access article distributed in accordance with the Creative Commons Attribution-NonCommercial-NoDerivs 4.0 International License (CC BY-NC-ND 4.0), which permits the noncommercial replication and distribution of the article with the strict proviso that no changes or edits are made and the original work is properly cited (including links to both the formal publication through the relevant DOI and the license). See: https://creativecommons.org/licenses/by-nc-nd/4.0/.

\section{References}

1. Bray F, Ferlay J, Soerjomataram I, et al. Global cancer 
statistics 2018: GLOBOCAN estimates of incidence and mortality worldwide for 36 cancers in 185 countries. CA Cancer J Clin 2018;68:394-424.

2. Harrison JM, Friese CR, Barton DL, et al. Heart Failure and Long-Term Survival Among Older Women With Breast Cancer. Oncol Nurs Forum 2018;45:A1-A11.

3. Abdelsalam M, Abu-Hegazy M, El-Hadaad HA, et al. Pathophysiology, Mechanism, and Outcome of Ischemic Stroke in Cancer Patients. J Stroke Cerebrovasc Dis 2020;29:105299.

4. Powell B, Bolton WD. Management of Lung Cancer with Concomitant Cardiac Disease. Thorac Surg Clin 2018;28:69-79.

5. Small HY, Montezano AC, Rios FJ, et al. Hypertension due to antiangiogenic cancer therapy with vascular endothelial growth factor inhibitors: understanding and managing a new syndrome. Can J Cardiol 2014;30:534-43.

6. Jang S, Zheng C, Tsai HT, et al. Cardiovascular toxicity after antiangiogenic therapy in persons older than 65 years with advanced renal cell carcinoma. Cancer 2016;122:124-30.

7. Chellappan DK, Leng KH, Jia LJ, et al. The role of bevacizumab on tumour angiogenesis and in the management of gynaecological cancers: A review. Biomed Pharmacother 2018;102:1127-44.

8. Kazazi-Hyseni F, Beijnen JH, Schellens JH. Bevacizumab. Oncologist 2010;15:819-25.

9. Coleman RL, Brady MF, Herzog TJ, et al. Bevacizumab and paclitaxel-carboplatin chemotherapy and secondary cytoreduction in recurrent, platinum-sensitive ovarian cancer (NRG Oncology/Gynecologic Oncology Group study GOG-0213): a multicentre, open-label, randomised, phase 3 trial. Lancet Oncol 2017;18:779-91.

10. Alter RA, White TG, Fanous AA, et al. Long-term benefit of intra-arterial bevacizumab for recurrent glioblastoma. J Exp Ther Oncol 2017;12:67-71.

11. Tewari KS, Sill MW, Penson RT, et al. Bevacizumab for advanced cervical cancer: final overall survival and adverse event analysis of a randomised, controlled, open-label, phase 3 trial (Gynecologic Oncology Group 240). Lancet 2017;390:1654-63.

12. Nakaya A, Kurata T, Yokoi T, et al. Retrospective analysis of bevacizumab-induced hypertension and clinical outcome in patients with colorectal cancer and lung cancer. Cancer Med 2016;5:1381-7.

13. Chen N, Ren M, Li R, et al. Bevacizumab promotes venous thromboembolism through the induction of PAI1 in a mouse xenograft model of human lung carcinoma.
Mol Cancer 2015;14:140.

14. Mallik R, Chowdhury TA. Metformin in cancer. Diabetes Res Clin Pract 2018;143:409-19.

15. Marrone KA, Zhou X, Forde PM, et al. A Randomized Phase II Study of Metformin plus Paclitaxel/Carboplatin/ Bevacizumab in Patients with Chemotherapy-Naive Advanced or Metastatic Nonsquamous Non-Small Cell Lung Cancer. Oncologist 2018;23:859-65.

16. Qu RN, Qu W. Metformin inhibits LPS-induced inflammatory response in VSMCs by regulating TLR4 and PPAR-gamma. Eur Rev Med Pharmacol Sci 2019;23:4988-95.

17. Tian R, Li R, Liu Y, et al. Metformin ameliorates endotoxemia-induced endothelial pro-inflammatory responses via AMPK-dependent mediation of HDAC5 and KLF2. Biochim Biophys Acta Mol Basis Dis 2019;1865:1701-12.

18. Hung CH, Chan SH, Chu PM, et al. Metformin regulates oxLDL-facilitated endothelial dysfunction by modulation of SIRT1 through repressing LOX-1-modulated oxidative signaling. Oncotarget 2016;7:10773-87.

19. Xie Z, Lin B, Jia X, et al. Enhanced IL-10 inhibits proliferation and promotes apoptosis of HUVECs through STAT3 signaling pathway in sepsis. Histol Histopathol 2021:18375.

20. Wang Y, Yang R, Yan F, et al. Medicarpin Protects Cerebral Microvascular Endothelial Cells Against OxygenGlucose Deprivation/Reoxygenation-Induced Injury via the PI3K/Akt/FoxO Pathway: A Study of Network Pharmacology Analysis and Experimental Validation. Neurochem Res 2021.

21. Wang Y, Che J, Zhao H, et al. Osthole alleviates oxidized low-density lipoprotein-induced vascular endothelial injury through suppression of transforming growth factor-beta1/Smad pathway. Int Immunopharmacol 2018;65:373-81.

22. Lee SJ, Lee I, Lee J, et al. Statins, 3-hydroxy-3methylglutaryl coenzyme A reductase inhibitors, potentiate the anti-angiogenic effects of bevacizumab by suppressing angiopoietin2, BiP, and Hsp90alpha in human colorectal cancer. Br J Cancer 2014;111:497-505.

23. Niu C, Chen Z, Kim KT, et al. Metformin alleviates hyperglycemia-induced endothelial impairment by downregulating autophagy via the Hedgehog pathway. Autophagy 2019;15:843-70.

24. Zheng A, Dubuis G, Ferreira CSM, et al. The PI3K/ Akt pathway is not a main driver in HDL-mediated cell protection. Cell Signal 2019;62:109347. 
25. Vanhoutte PM, Shimokawa H, Feletou M, et al. Endothelial dysfunction and vascular disease - a 30th anniversary update. Acta Physiol (Oxf) 2017;219:22-96.

26. Kapelakis I, Toutouzas K, Drakopoulou M, et al. Bevacizumab increases the incidence of cardiovascular events in patients with metastatic breast or colorectal cancer. Hellenic J Cardiol 2017;58:215-19.

27. Ngo DTM, Williams T, Horder S, et al. Factors Associated with Adverse Cardiovascular Events in Cancer Patients Treated with Bevacizumab. J Clin Med 2020;9:2664.

28. Hauge A, Gaustad JV, Huang R, et al. DCE-MRI and Quantitative Histology Reveal Enhanced Vessel Maturation but Impaired Perfusion and Increased Hypoxia in Bevacizumab-Treated Cervical Carcinoma. Int J Radiat Oncol Biol Phys 2019;104:666-76.

29. Sirtori CR, Franceschini G, Gianfranceschi G, et al. Metformin improves peripheral vascular flow in nonhyperlipidemic patients with arterial disease. J Cardiovasc Pharmacol 1984;6:914-23.

30. Gou L, Liu G, Ma R, et al. High fat-induced inflammation in vascular endothelium can be improved by Abelmoschus esculentus and metformin via increasing the expressions of miR-146a and miR-155. Nutr Metab (Lond) 2020;17:35.

31. Wang JC, Li GY, Wang B, et al. Metformin inhibits metastatic breast cancer progression and improves chemosensitivity by inducing vessel normalization via PDGF-B downregulation. J Exp Clin Cancer Res 2019;38:235.

32. Bakhashab S, Ahmed F, Schulten HJ, et al. Proangiogenic Effect of Metformin in Endothelial Cells Is via Upregulation of VEGFR1/2 and Their Signaling under Hyperglycemia-Hypoxia. Int J Mol Sci 2018;19:293.

33. Day EA, Ford RJ, Smith BK, et al. Metformin-induced increases in GDF15 are important for suppressing appetite and promoting weight loss. Nat Metab 2019;1:1202-08.

34. Au Yeung SL, Luo S, Schooling CM. The impact of GDF15 , a biomarker for metformin, on the risk of coronary

Cite this article as: Chen L, Yin Y, Liu G. Metformin alleviates bevacizumab-induced vascular endothelial injury by up-regulating GDF15 and activating the PI3K/AKT/FOXO/ PPAR $\gamma$ signaling pathway. Ann Transl Med 2021;9(20):1547. doi: 10.21037/atm-21-4764 artery disease, breast and colorectal cancer, and type 2 diabetes and metabolic traits: a Mendelian randomisation study. Diabetologia 2019;62:1638-46.

35. Qiang P, Shao Y, Sun YP, et al. Metformin inhibits proliferation and migration of endometrial cancer cells through regulating PI3K/AKT/MDM2 pathway. Eur Rev Med Pharmacol Sci 2019;23:1778-85.

36. Arunachalam G, Samuel SM, Marei I, et al. Metformin modulates hyperglycaemia-induced endothelial senescence and apoptosis through SIRT1. Br J Pharmacol 2014;171:523-35.

37. Gunduz D, Troidl C, Tanislav C, et al. Role of PI3K/ Akt and MEK/ERK Signalling in cAMP/Epac-Mediated Endothelial Barrier Stabilisation. Front Physiol 2019;10:1387.

38. Liu Y, Tie L. Apolipoprotein M and sphingosine-1phosphate complex alleviates TNF-alpha-induced endothelial cell injury and inflammation through PI3K/ AKT signaling pathway. BMC Cardiovasc Disord 2019;19:279.

39. Li CG, Mahon C, Sweeney NM, et al. PPARgamma Interaction with UBR5/ATMIN Promotes DNA Repair to Maintain Endothelial Homeostasis. Cell Rep 2019;26:1333-43 e7.

40. Ha G, De Torres F, Arouche N, et al. GDF15 secreted by senescent endothelial cells improves vascular progenitor cell functions. PLoS One 2019;14:e0216602.

41. Gao F, Artham S, Sabbineni H, et al. Akt1 promotes stimuli-induced endothelial-barrier protection through FoxO-mediated tight-junction protein turnover. Cell Mol Life Sci 2016;73:3917-33.

42. Chen Y, Wang Y, Jiang Y, et al. High-glucose treatment regulates biological functions of human umbilical vein endothelial cells via Sirt1/FOXO3 pathway. Ann Transl Med 2019;7:199.

(English Language Editor: A. Kassem) 

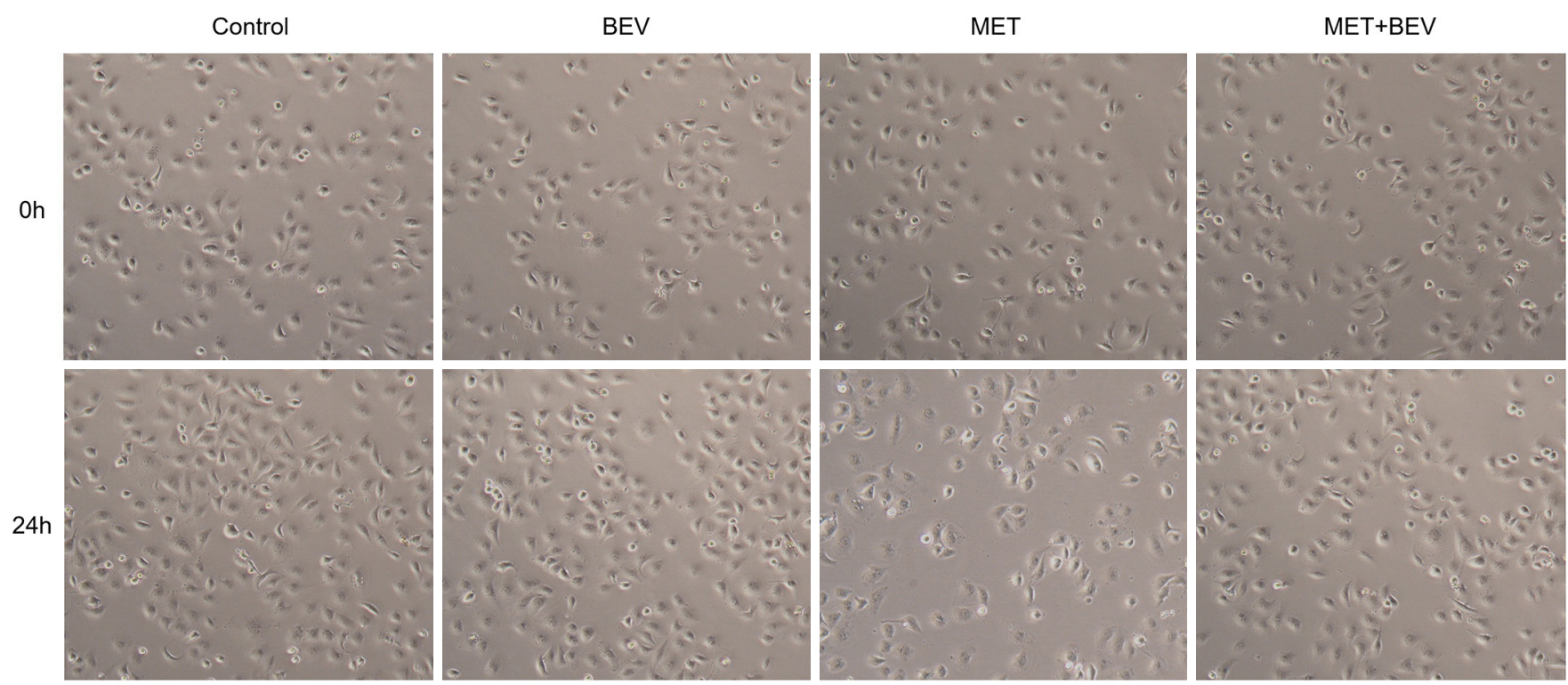

Figure S1 Representative images of HUVECs before and after treatment under the brightfield $(\times 200)$. HUVECs, human umbilical vein endothelial cells; MET, metformin; BEV, bevacizumab. 